Jin-Long Yan*

\title{
Crystal structure of diethanol- $\mathrm{K}^{1} \mathrm{O}$-bis $\left(\mu_{2}-\mathrm{N}-((2\right.$-oxidonaphthalen-1-yl) methylene)pyrazine-2-carbohydrazonato- $\mathrm{K}^{5} \mathrm{~N}, \mathrm{O}, \mathrm{O}^{\prime}: \mathrm{O}^{\prime}: \mathrm{N}^{\prime}$ )-bis(nitrato- $\mathrm{K}^{2} \mathrm{O}$, $O^{\prime}$ )dieuropium(III), $\mathrm{C}_{36} \mathrm{H}_{32} \mathrm{~N}_{10} \mathrm{O}_{12} \mathrm{Eu}_{2}$
}

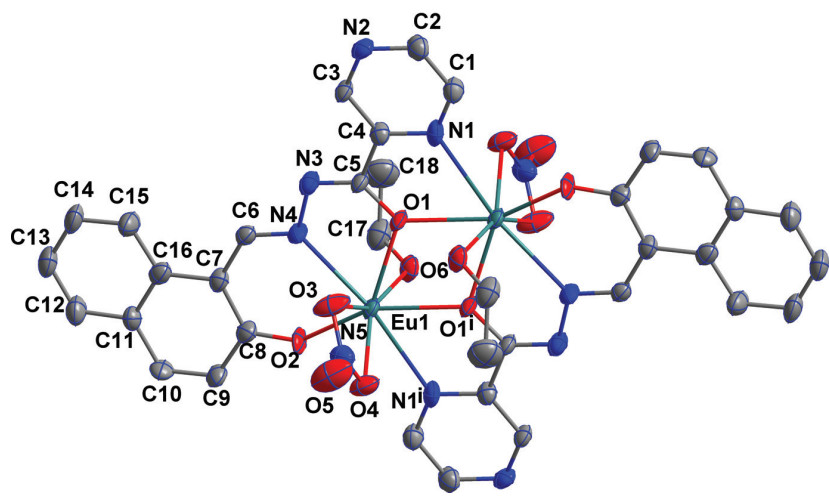

https://doi.org/10.1515/ncrs-2019-0772

Received October 17, 2019; accepted December 31, 2019; available online January 21, 2020

\begin{abstract}
$\mathrm{C}_{36} \mathrm{H}_{32} \mathrm{~N}_{10} \mathrm{O}_{12} \mathrm{Eu}_{2}$, monoclinic, $C 2 / c$ (no. 15), $a=16.445(2) \AA$, $b=10.0920(14) \AA, \quad c=23.865(3) \AA, \quad \beta=100.115(8)^{\circ}$, $V=3899.3(9) \AA^{3}, Z=4, R_{\mathrm{gt}}(F)=0.0687, w R_{\mathrm{ref}}\left(F^{2}\right)=0.2065$, $T=150(2) \mathrm{K}$.
\end{abstract}

\section{CCDC no.: 1974929}

The dinuclear title complex is shown in the figure. Table 1 contains crystallographic data and Table 2 contains the list of the atoms including atomic coordinates and displacement parameters.

\section{Source of material}

The hydrazone ligand, $N$-((2-hydroxynaphthalen-1yl)methylene)pyrazine-2-carbohydrazonic acid $\left(\mathrm{H}_{2} \mathrm{~L}\right)$ was prepared via the condensation of 2-pyrazinoylhydrazide and 2-hydroxy-1-naphthaldehyde according to the literature [4]. The reaction of europium(II) nitrate in ethanol under basic conditions yielded the title compound. The crystals of the title complex were obtained by slow evaporation of the ethanol solution.

*Corresponding author: Jin-Long Yan, School of Science and Technology, Jiaozuo Teachers College, Jiaozuo 454001, P.R. China, e-mail: jinlong_yan@163.com. https://orcid.org/0000-0003-2396-1499
Table 1: Data collection and handling.

\begin{tabular}{ll}
\hline Crystal: & Plate, red \\
Size: & $0.10 \times 0.06 \times 0.02 \mathrm{~mm}$ \\
Wavelength: & Cu $K \alpha$ radiation $(1.54178 \AA)$ \\
$\mu:$ & $23.45 \mathrm{~mm}^{-1}$ \\
Diffractometer, scan mode: & Bruker APEX-II, $\varphi$ and $\omega$-scans \\
$\theta_{\max }$, completeness: & $74.8^{\circ},>98 \%$ \\
$N\left(h k l_{\text {measured }}, N\left(h k l l_{\text {unique }}, R_{\text {int }}:\right.\right.$ & $18243,3918,0.094$ \\
Criterion for $I_{\text {obs }}, N(h k l)_{\text {gt }}:$ & $I_{\text {obs }}>2 \sigma\left(I_{\text {obs }}\right), 3025$ \\
$N(\text { param })_{\text {refined }}:$ & 273 \\
Programs: & Bruker programs [1], SHELX [2] \\
\hline
\end{tabular}

Table 2: Fractional atomic coordinates and isotropic or equivalent isotropic displacement parameters $\left(\AA^{2}\right)$.

\begin{tabular}{lrrrr}
\hline Atom & $\boldsymbol{x}$ & $\boldsymbol{y}$ & $\boldsymbol{z}$ & $\boldsymbol{U}_{\text {iso }} / \boldsymbol{U}_{\text {eq }}$ \\
\hline Eu1 & $0.51082(3)$ & $0.67657(6)$ & $0.46830(2)$ & $0.0254(2)$ \\
O1 & $0.5489(4)$ & $0.4530(7)$ & $0.4663(3)$ & $0.0288(15)$ \\
O2 & $0.5135(5)$ & $0.8478(7)$ & $0.4134(3)$ & $0.0314(17)$ \\
O3 & $0.6558(5)$ & $0.6695(9)$ & $0.5172(4)$ & $0.050(2)$ \\
O4 & $0.5763(5)$ & $0.8026(9)$ & $0.5529(4)$ & $0.044(2)$ \\
O5 & $0.7049(6)$ & $0.7785(13)$ & $0.5941(5)$ & $0.073(3)$ \\
O6 & $0.3891(4)$ & $0.5946(8)$ & $0.4056(3)$ & $0.0324(17)$ \\
H6 & 0.3435 & 0.5827 & 0.4161 & $0.049 *$ \\
N1 & $0.6057(5)$ & $0.2080(9)$ & $0.4863(4)$ & $0.0283(19)$ \\
N2 & $0.7337(5)$ & $0.1093(9)$ & $0.4364(4)$ & $0.032(2)$ \\
N3 & $0.6309(5)$ & $0.4872(10)$ & $0.3987(4)$ & $0.032(2)$ \\
N4 & $0.5948(5)$ & $0.6144(9)$ & $0.3949(4)$ & $0.0291(19)$ \\
N5 & $0.6476(5)$ & $0.7522(10)$ & $0.5564(4)$ & $0.036(2)$ \\
C1 & $0.6341(7)$ & $0.0872(11)$ & $0.4970(5)$ & $0.034(2)$ \\
H1 & 0.6105 & 0.0332 & 0.5225 & $0.041^{*}$ \\
C2 & $0.6978(6)$ & $0.0362(12)$ & $0.4722(5)$ & $0.035(2)$ \\
H2 & 0.7162 & -0.0519 & 0.4807 & $0.042^{*}$ \\
C3 & $0.7035(6)$ & $0.2300(11)$ & $0.4240(4)$ & $0.027(2)$ \\
H3 & 0.7263 & 0.2828 & 0.3976 & $0.032^{*}$ \\
C4 & $0.6391(6)$ & $0.2813(10)$ & $0.4486(4)$ & $0.026(2)$ \\
C5 & $0.6054(6)$ & $0.4177(11)$ & $0.4363(4)$ & $0.028(2)$ \\
C6 & $0.6192(6)$ & $0.6886(10)$ & $0.3561(4)$ & $0.026(2)$ \\
H6A & 0.6551 & 0.6475 & 0.3342 & $0.032^{*}$ \\
C7 & $0.5988(6)$ & $0.8226(11)$ & $0.3427(4)$ & $0.028(2)$ \\
C8 & $0.5473(6)$ & $0.8989(11)$ & $0.3725(5)$ & $0.030(2)$ \\
C9 & $0.5308(6)$ & $1.0341(11)$ & $0.3577(5)$ & $0.032(2)$ \\
H9 & 0.4954 & 1.0834 & 0.3774 & $0.039^{*}$ \\
C10 & $0.5640(7)$ & $1.0947(11)$ & $0.3163(5)$ & $0.033(2)$ \\
H10 & 0.5532 & 1.1859 & 0.3086 & $0.040^{*}$ \\
C11 & $0.6151(6)$ & $1.0230(11)$ & $0.2841(4)$ & $0.028(2)$ \\
& & & &
\end{tabular}


Table 2 (continued)

\begin{tabular}{lrrrr}
\hline Atom & $\boldsymbol{x}$ & $\boldsymbol{y}$ & $\boldsymbol{z}$ & $\boldsymbol{U}_{\text {iso }} \boldsymbol{U}_{\text {eq }}$ \\
\hline C12 & $0.6465(8)$ & $1.0820(13)$ & $0.2390(5)$ & $0.044(3)$ \\
H12 & 0.6347 & 1.1729 & 0.2308 & $0.052^{*}$ \\
C13 & $0.6927(7)$ & $1.0157(13)$ & $0.2067(5)$ & $0.040(3)$ \\
H13 & 0.7107 & 1.0574 & 0.1754 & $0.048^{*}$ \\
C14 & $0.7130(8)$ & $0.8859(14)$ & $0.2205(5)$ & $0.045(3)$ \\
H14 & 0.7479 & 0.8392 & 0.1996 & $0.055^{*}$ \\
C15 & $0.6834(8)$ & $0.8219(12)$ & $0.2645(5)$ & $0.043(3)$ \\
H15 & 0.6976 & 0.7317 & 0.2724 & $0.052^{*}$ \\
C16 & $0.6334(6)$ & $0.8870(12)$ & $0.2972(5)$ & $0.033(2)$ \\
C17 & $0.3786(8)$ & $0.5830(14)$ & $0.3446(5)$ & $0.046(3)$ \\
H17A & 0.4099 & 0.6545 & 0.3296 & $0.056^{*}$ \\
H17B & 0.3194 & 0.5948 & 0.3281 & $0.056^{*}$ \\
C18 & $0.4071(10)$ & $0.4527(16)$ & $0.3263(6)$ & $0.063(4)$ \\
H18A & 0.4009 & 0.4508 & 0.2847 & $0.095^{*}$ \\
H18B & 0.3738 & 0.3818 & 0.3389 & $0.095^{*}$ \\
H18C & 0.4653 & 0.4397 & 0.3431 & $0.095^{*}$ \\
\hline
\end{tabular}

\section{Experimental details}

The structure was solved by direct methods and refined with the SHELX crystallographic software package [2]. The hydrogen atoms were placed at calculated positions and refined as riding atoms with isotropic displacement parameters.

\section{Discussion}

Many hydrazones and their complexes have been found to show promising biological activities [3]. Particularly, the dysprosium(III) complexes of hydrazones derived from 2pyrazinoylhydrazide and subsituted salicylaldehyde show distinct single-molecule-magnet behaviour [4, 5]. In this work, the europium(III) complex of a pyrazinoylhydrazone ligand was synthesized and characterized by X-ray diffraction.

As shown in the figure, there exists one discrete dimeric $\mathrm{Eu}$ (II) complex located around a center of inversion with the Eu-Eu distance of 3.9123(5) $\AA$ in the title crystal structure. Each europium(III) ion is eight-coordinated to one $\mathrm{O}_{2} \mathrm{~N}$ donor set from one $L^{2-}$, one $\mathrm{N}$ atom and one $\mu^{2}-\mathrm{O}$ atom from the adjacent $L^{2-}$ ligand (Symmetry code: (i) $-x+1,-y+1$, $-z+1$ ), one $\mathrm{O}$ atom from one coordinated ethanol and two $\mathrm{O}$ atoms from one nitrate anion showing a bidentated coordination mode. These coordinations result in a distorted bicapped triangular prism coordination geometry. In the solid, pairs of intermolecular $\mathrm{O}-\mathrm{H} \cdots \mathrm{N}$ hydrogen bonds between ethanol and terminal pyrazine $\mathrm{N}$ atom link dimers into an one-dimensional chain.

\section{References}

1. Bruker. SMART and SAINT. Bruker AXS Inc., Madison, WI, (USA) 2007.

2. Sheldrick, G. M.: Crystal structure refinement with SHELX. Acta Crystallogr. C71 (2015) 3-8.

3. Ye, X.-P.; Wang, G.-J.; Pan, P.; Zhang, Z.-P.; Wu, W.-N.; Wang, Y.: Syntheses, Crystal structures and biological activities of two $\mathrm{Cu}$ (II) complexes with an acylhydrazone ligand bearing pyrrole unit. Chin. J. Inorg. Chem. 30 (2014) 2789-2795.

4. Tian, H.-Q.; Zhao, L.; Guo, Y.-N.; Tang, J.-K.; Liu, Z.-L.: Quadruple$\mathrm{CO}_{3}{ }^{2}$-bridged octanuclear dysprosium(III) compound showing single-molecule magnet behaviour. Chem. Commun. 48 (2012) 708-710.

5. Tian, H.-Q.; Guo, Y.-N.; Zhao, L.; Tang, J.-K.; Liu, Z.-L.: Hexanuclear dysprosium(III) compound incorporating vertex- and ddge-sharing Dy ${ }_{3}$ triangles exhibiting single-molecule-magnet behavior. Inorg. Chem. 50 (2011) 8688-8690. 